\title{
AN OSCILLATION CRITERION FOR INHOMOGENEOUS STIELTJES INTEGRO-DIFFERENTIAL EQUATIONS
}

\author{
M.A. EL-SAYED \\ Department of Mathematics \\ Faculty of Science \\ Cairo University, Giza \\ Egypt \\ (Received September 28, 1993 and in revised form February 26, 1993)
}

ABSTRACT. The aim of the paper is to give an oscillation theorem for inhomogeneous Stieltjes integro-differential equation of the form $p(t) x^{\prime}+\int_{a}^{t} x(s) d \sigma=$ $f(t)$. The paper generalizes the author's work [2].

KEYWORDS AND PHRASES: Oscillation theory, Inhomogeneous linear equation, Stieltjes integro-differential equation.

1992 AMS SUBJECT CLASSIFICATION CODES. 45J05, 45D05, 34C10, 39A10.

\section{INTRODUCTION}

We are concerned here with the oscillation behaviour of the inhomogeneous integro-differential equation

$$
p(t) x^{\prime}+\int_{a}^{t} x(s) d \sigma f(t), \quad a, t \in I=[0, \infty[,
$$

where $f, p, \sigma \in B V_{1 \circ c}(I)$ are real-valued right-continuous functions, $p(t)>0$ and

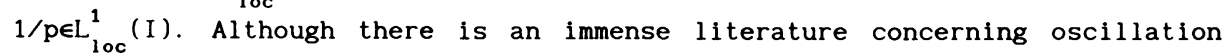
criteria of second order homogeneous ordinary differential equations, a little is known about the oscillation of equation (1), even when $f^{\prime}, \sigma \in C^{(1)}(I)$ and $\sigma^{\prime}$ is oscillatory [6]. The latter corresponds to the second order ordinary inhomogeneous differential equation

$$
\left(p(t) x^{\prime}\right)^{\prime}+q(t) x=g(t), t \in I
$$

where $g=f^{\prime}, q=\sigma^{\prime}$ are oscillatory functions. This case has been studied in [2].

The advantage of studying (1), instead of (2), is the flexibility to handle ordinary linear inhomogeneous differential equations and ordinary difference equations, as well as equations of mixed type, within the frame of a unique theory (see $[3,4])$.

An oscillation criterion for a special case of equation (1) ( $v i z$. $f \equiv 0$ ) is given in [4, Theorem 4.3.5 page 83]. The theorem is an extension of the wellknown Fite-Wintener-Leighton theorem. The method used involves the integration of the function $p$ on the whole interval $[0, \infty[$ and assumes that $\sigma(t) \rightarrow \infty$ as $t \rightarrow \infty$.

The method used here to study the oscillation behaviour of equation (1) depends on an extension of a comparison theorem of Sturm's type due to 
Leighton [4, Theorem 1.1.0, p. 5] (see also [5]).

The main results of the paper are formulated in theorems 1 and 2 of section 3, which generalize theorems 3.1 and 3.2 of [2] respectively. In section 2 a necessary background for the study, carried out in section 3 , is provided.

\section{PRELIMINARIES.}

In the sequel, we assume that all functions under consideration are realvalued functions defined on the semi-open semi-infinite interval $I=[0, \infty[$. As usual, $\mathrm{BV}_{10 \mathrm{c}}$ (I) denotes the space of functions, locally of bounded variations over I and $\mathrm{AC}_{10 \mathrm{C}}(\mathrm{I})$ - the space of locally absolutely continuous functions defined on $I$. The functions $\sigma, f, p \in B V_{10 c}$ (I) are assumed to be right-continuous and, for the sake of simplicity, have a finite number of discontinuities in every finite subinterval.

By a solution of (1) is meant a real-valued function $x \in A C_{10 c}$ (I) with the property that $\mathrm{px}^{\prime} \in \mathrm{BV}_{10 \mathrm{c}}(\mathrm{I})$ and $\mathrm{x}$ satisfies (1) almost everywhere (a.e.) on $I$. For the existence and uniqueness results for equation (1), we refer to [4, Theorem I.3.1, p. 265 and Theorem I.3.2, p. 269] (see also [1]).

Let $[a, b]$ be a compact subinterval in $I$. In addition to the above stated assumptions, let $p$ and $\sigma$ be continuous at the end points. Defining the quadratic functional $Q[u]$ with domain

by

$$
\mathfrak{D}_{\mathrm{Q}}=\left\{\mathrm{u}: u \in A C_{10 c}[a, b], p u \in B V_{10 c}[a, b], u(a)=u(b)=0\right\}
$$

we prove the following

$$
Q[u]=\int_{a}^{b}\left(p u^{\prime 2} d t-u^{2} d \sigma\right)
$$

LEMMA 1. Let $f$ be a nonincreasing (nondecreasing) function in $[a, b]$. If there exists a function $u \in \mathbb{D}_{Q}$, not identically zero, such that $Q[u] \leq 0$, then there is no solution of (1) which is positive (negative) in ]a,b[, unless it is a constant multiple of $u$.

PROOF. Suppose to the contrary that there exists a positive solution $v$ of (1) in ] $a, b\left[\right.$. For all such $v$, we have $p v^{\prime} / v \in B V_{10 c}(a, b)$. . Let $a<\alpha<\beta<b$. For $u \in D_{0}$ the integral

$$
\int_{\alpha}^{\beta} u^{2} d\left(p v^{\prime} / v\right)
$$

exists.

Now

$$
\begin{aligned}
\int_{\alpha}^{\beta} u^{2} d\left(p v^{\prime} / v\right) & =\int_{\alpha}^{\beta}\left[u^{2} d\left(p v^{\prime}\right) / v-p\left(u v^{\prime} / v\right)^{2}\right] \\
& =\int_{\alpha}^{\beta} u^{2} \frac{d f}{v}-\int_{\alpha}^{\beta} u^{2} d \sigma-\int_{\alpha}^{\beta} p\left(u v^{\prime} / v\right)^{2} .
\end{aligned}
$$

On the other hand,

Hence

$$
\int_{\alpha}^{\beta} u^{2} d\left(p v^{\prime} / v\right)=u^{2} p v^{\prime} /\left.v\right|_{\alpha} ^{\beta}-2 \int_{\alpha}^{\beta} p u u^{\prime} v^{\prime} / v .
$$

$$
\int_{\alpha}^{\beta} p u^{\prime 2}-u^{2} d \sigma=u^{2} p v^{\prime} /\left.v\right|_{\alpha} ^{\beta}+\int_{\alpha}^{\beta} p v^{2}\left[(u / v)^{\prime}\right]^{2}-\int_{\alpha}^{\beta} u^{2} \frac{d f}{v}
$$


If $v(a) \neq 0$ and $v(b) \neq 0$, then we can take the limit as $\alpha \rightarrow a+0, \beta \rightarrow b-0$ and get

$$
Q[u]=\int_{a}^{b} p v^{2}\left[(u / v)^{\prime}\right]^{2}-\int_{a}^{b} u^{2} \frac{d f}{v} \geq \int_{a}^{b} p v^{2}\left[(u / v)^{\prime}\right]^{2} \geq 0 .
$$

The hypothesis on $u$ implies that $Q[u]=0$. Since $v \neq 0$, we have $(u / v)^{\prime}=0$. Therefore $u$ is a constant multiple of $v$. The latter cannot occur since $u(a)=0$ but $v(a) \neq 0$. The contradiction shows that $v$ is not positive.

Now consider the case $v(a)=v(b)=0$. This implies that neither $v^{\prime}(a)$ nor $v^{\prime}$ (b) vanish. Hence

$$
\lim _{\alpha \rightarrow a+0} \frac{u^{2}(\alpha) p(\alpha) v^{\prime}(\alpha)}{v(\alpha)} p(a) v^{\prime} \lim _{\alpha \rightarrow a+0} \frac{u^{2}(\alpha)}{v(\alpha)}
$$

provided that the latter limit exists. The hypothesis on $\sigma$ implies that it is continuous in some right-neighbourhood of $a$. Thus $p(t) v^{\prime}(t)$ is continuous in such a neighbourhood. Similarly $p(t)$ is continuous in some, possibly different, right-neighbourhood of a. Hence $v^{\prime}$ is continuous (i.e. is an ordinary derivative) in some right-neighbourhood $] a, a+\delta[, \delta>0$.

In the same way it can be shown that $u^{\prime}$ is an ordinary derivative in ]a, $a+\eta[, \eta>0$. Thus in ] $a, a+\eta[$,

$$
\left[u^{2}(t)\right]^{\prime}=2 u(t) u^{\prime}(t) .
$$

Since $u, v \in A C{ }_{10 c}[a, b]$, we may apply L'Hopital's rule to the limit in the right hand side of (4) to obtain

$$
\lim _{\alpha \rightarrow a+0} \frac{u^{2}(t)}{v(\alpha)}=\lim _{\alpha \rightarrow a+0} \frac{2 u(\alpha) u^{\prime}(\alpha)}{v^{\prime}(\alpha)}=0 .
$$

Similarly it can be shown that

$$
\lim _{\beta \rightarrow b-0} \frac{u^{2}(\beta) p(\beta) v^{\prime}(\beta)}{v(\beta)}=0 .
$$

Letting $\alpha \rightarrow a+0$ and $\beta \rightarrow b-0$ in (3), we obtain $Q[u] \geq 0$ and thus derive a contradiction, unless $v=c u$.

In the mixed case when one of $v(a)$ or $v(b)$ is zero, while the other is not, it is clear from the foregoing proof that $Q[u] \geq 0$ still holds. This completes the proof.

In addition to the quadratic functional $Q[u]$ we define the quadratic functional $\tilde{Q}[u]$ on the domain

by

$$
\tilde{D}_{\tilde{Q}}=\left\{u: u \in A C[a, b], \tilde{p} u^{\prime} \in B V[a, b], u(a)=u(b)=0\right\}
$$

$$
\tilde{Q}[u]=\int_{a}^{b} \tilde{p} u^{\prime 2}-u^{2} d v
$$

which is associated with the homogeneous Stieltjes integro-differential equation

$$
\tilde{p}(t) w^{\prime}+\int_{a}^{t} w(s) d v(s)=c
$$

where $\tilde{p}, v \in B V_{10 c}(I)$ are real-valued right-continuous functions, $\tilde{p}(t)>0$ on $I$ with $1 / \tilde{p} \in L_{1 \circ c}^{1}$ (I) and $c$ is a real constant.

Defining

$V[u]=\tilde{Q}[u]-Q[u]$,

we have the following 
LEMMA 2. Let the conditions of the above lemma be satisfied. If there exists a nontrivial solution $u \in \mathfrak{I}$ of (3) such that $v[u] \geq 0$, then there is no solution of (1) which is positive (negative) in ]a,bl, unless it is a constant multiple of $u$.

PROOF. Let $u$ be a solution of (3) that satisfies $u(a)=u(b)=0$. Then

$$
\int_{a}^{b} u d\left(\tilde{p} u^{\prime}\right)=\left.\tilde{p} u u^{\prime}\right|_{a} ^{b}-\int_{a}^{b} \tilde{p} u^{\prime 2}=-\int_{a}^{b} u^{2} d v .
$$

Hence $\tilde{Q}[u]=0$. Therefore $V[u] \geq 0$ implies that $Q[u] \leq 0$. Now the lemma follows from lemma 1.

3. AN OSCILLATION CRITERION.

In this section we state and prove the main theorem of the paper on the oscillation of the inhomogeneous Stieltjes integro-differential equation (1).

THEOREM 1. Let there exist two positive increasing divergent sequences $\left\{a_{n}^{+}\right\},\left\{a_{n}^{-}\right\}$and two sequences of positive numbers $\left\{c_{n}^{+}\right\},\left\{c_{n}^{-}\right\}$such that

$$
\begin{aligned}
v_{n}^{ \pm}=\int_{a_{n}^{ \pm}}^{a_{n}^{ \pm}+\pi / \sqrt{c_{n}^{ \pm}}} & \left(c_{n}^{ \pm}[1-p(t)] \cos ^{2}\left\{\sqrt{c_{n}^{ \pm}}\left(t-a_{n}^{ \pm}\right)\right\} d t\right. \\
& \left.+\sin ^{2}\left\{\sqrt{c_{n}^{ \pm}\left(t-a_{n}^{ \pm}\right)}\right\}\left[d \sigma(t)-c_{n}^{ \pm} d t\right]\right) \geq 0
\end{aligned}
$$

for every $n \in \mathbb{N}$. Assume that the function $f$ is nondecreasing in $\left[a_{n}^{+}, a_{n}^{+}+\pi / \sqrt{c_{n}^{+}}\right]$ and nonincreasing in $\left[a_{n}^{-}, a_{n}^{-}+\pi / \sqrt{c_{n}^{-}}\right]$and $\sigma, p$ are continuous at $a_{n}^{ \pm}, a_{n}^{ \pm}+\pi / \sqrt{c_{n}^{ \pm}}$ for every $n \in \mathbb{N}$. Then equation (1) is oscillatory.

PROOF. If we suppose, to the contrary, that (1) has an eventually positive solution, then there exists $n_{0} \in \mathbb{N}$ such that $y(t)>0 \forall t \geq n_{0}$ and $f$ is nonincreasing in the intervals $\left[a_{n}^{-}, a_{n}^{-}+\pi / \sqrt{c_{n}^{-}}\right] \forall n \geq n_{0}$.

Consider the linear homogeneous differential equation

$$
x^{\prime \prime}(t)+c_{n}^{-} x(t)=0, \quad t \in\left[a_{n}^{-}, a_{n}^{-}+\pi / \sqrt{c_{n}^{-}}\right], n \geq n_{0} \text {. }
$$

This equation has the solution $u(t)=\sin \left\{\sqrt{c_{n}^{-}}\left(t-a_{n}^{-}\right)\right\}$, which has two consecutive zeros at $t=a_{n}^{-}$and at $t=a_{n}^{-}+\pi / \sqrt{c_{n}^{-}}$. Therefore, by lemma 2 y cannot be positive in $\int a_{n}^{-}, a_{n}^{-}+\pi / \sqrt{c_{n}^{-}}$unless $y$ is a constant multiple of $\sin \left\{\sqrt{c_{n}^{-}}\left(t-a_{n}^{-}\right)\right\}$. Both cases lead to a contradiction.

Next, suppose that the solution is eventually negative. We use the fact that $f$ is nondecreasing in $\left[a_{n}^{+}, a_{n}^{+}+\pi / \sqrt{c_{n}^{+}}\right]$and $y<0$ for all $n$ greater than or equal to some $n_{0}$ to get the desired contradiction.

In the case of unforced equations, i.e. $f \equiv 0$, we have the following theorem, which is a special case of Theorem 1.

THEOREM 2. If there exists an increasing divergent sequence of positive numbers $\left\{a_{n}\right\}$ and a sequence of positive numbers $\left\{c_{n}\right\}$ such that

$$
\begin{aligned}
v_{n}=\int_{a_{n}}^{a_{n}+\pi / \sqrt{c_{n}}}\left(c_{n}\right. & 11-p(t)] \cos ^{2}\left\{\sqrt{c_{n}}\left(t-a_{n}\right)\right\} d t \\
& \left.+\sin ^{2}\left\{\sqrt{c_{n}}\left(t-a_{n}\right)\right\}\left[d \sigma(t)-c_{n} d t\right]\right) \geq 0 .
\end{aligned}
$$


then the equation

$$
p(t) x^{\prime}+\int_{a}^{t} x(s) d \sigma=0, \quad a, t \in I=[0, \infty[,
$$

is oscillatory.

REMARK. Setting $f, \sigma \in C^{(1)}(I), \sigma(t)=\int_{0}^{t} q(s) d s, \quad q \in L_{1 \circ c}^{1}$ (I) and $f^{\prime}=g$, theorems 1 and 2 stated above are reduced immediately to theorems 3.1 and 3.2 of [2] correspondingly.

EXAMPLE. Let $a \in]-\infty, \infty[$ and let $b, c \in[0, \infty[$ such that $a+b \geq 1$. Define on $[\pi / 2, \infty[$ a function $f$ as follows. For $t \in[(n-1 / 2) \pi,(n+1 / 2) \pi[, n \in \mathbb{N}$ we set

$$
f(t)= \begin{cases}t^{c}[t-(n-1 / 2) \pi], & \text { if } n \text { is odd, } \\ t^{c}[(n-1 / 2) \pi-t], & \text { if } n \text { is even. }\end{cases}
$$

Let $\sigma(t)=a t+b \sin 2 t$. Then, the inhomogeneous integro-differential equation

is oscillatory.

$$
x^{\prime}+\int_{a}^{t} x(s) d \sigma=f(t)
$$

PROOF. Setting $p(t) \equiv 1, c_{n} \equiv 1, a_{n}^{+}=(2 n-1 / 2) \pi$ and $a_{n}^{-}(2 n-3 / 2) \pi$ in $(6)$, we get

$$
v_{n}^{ \pm}=\pi(a+b-1) / 2 \geq 0
$$

Noting that $f$ is right-continuous and increasing in the interval $\left[a_{n}^{+}, a_{n}^{+}+\pi[\right.$ and decreasing in $\left[a_{n}^{-}, a_{n}^{-}+\pi[\right.$, and applying Theorem 1 , we get the required.

It is remarkable that, results of [2] cannot be applied to this example. This is due to the fact that $f$ is not continuous. Also, as a special case, if $a=-1$ and $b \geq 2$ or $a=0$ and $b \geq 1$, we see that $1 \mathrm{im} \sigma(t)=-\infty$ in the first case and $1 \mathrm{im}$ $\sigma(t)$ does not exist in the second case as $t \rightarrow \infty$. Therefore, the criterion of [3] cannot be applied here either.

\section{REFERENCES}

1. F. V. ATKINSON, Discrete and continuous boundary problems, Academic Press, New York (1964).

2. M. A. EL-SAYED, An Oscillation criterion for forced second order linear differential equation, Proc. Amer. Math. Soc. (To appear).

3. A. B. MINGARELLI, Volterra-Stieltjes integral equations and generalized ordinary differential expressions, Springer-Verlag, New York, Lecture Notes in Mathematics no. 989 (1983).

4. A. B. MINGARELLI and S. G. HALVORSEN, Non-Oscillation Domains of Differential equations with two parameters, Springer-Verlag, New York, Lecture Notes in Mathematics no. 1338 (1988).

5. C. A. SWANSON, Comparison and oscillation theory of linear differential equations, Academic Press, 1968.

6. J. S. W. WONG, Second order nonlinear forced oscillations, SIAM J. Math. Anal., vol. 19, No. 3(1988), 667-675. 


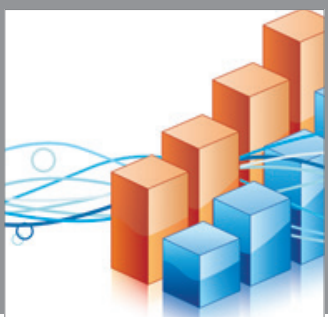

Advances in

Operations Research

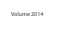

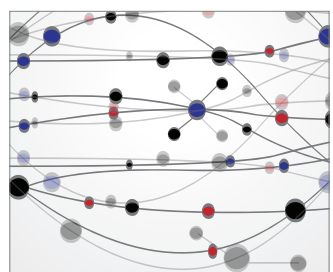

\section{The Scientific} World Journal
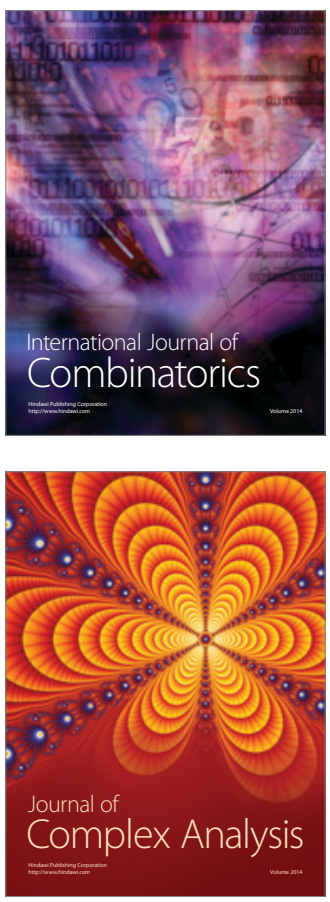

International Journal of

Mathematics and

Mathematical

Sciences
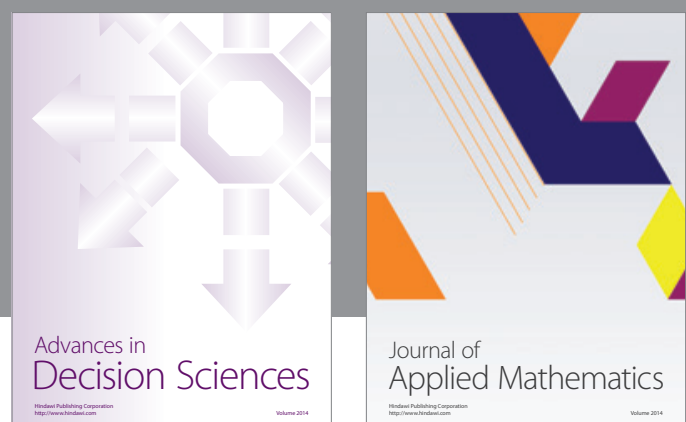

Journal of

Applied Mathematics
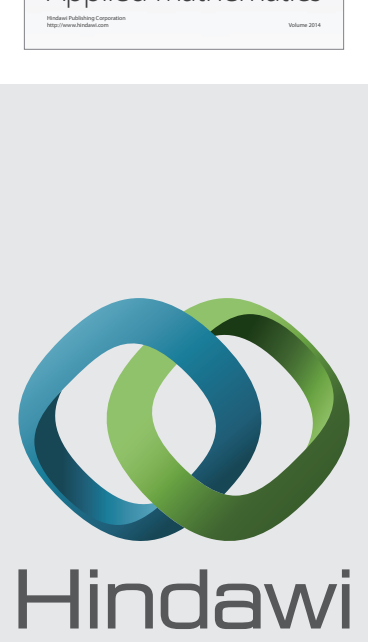

Submit your manuscripts at http://www.hindawi.com
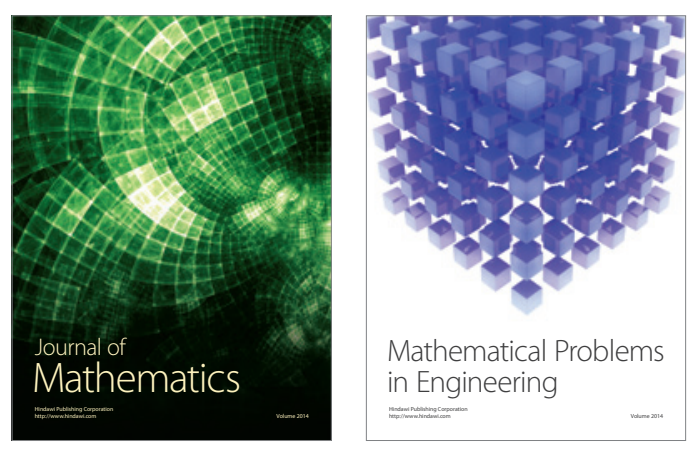

Mathematical Problems in Engineering
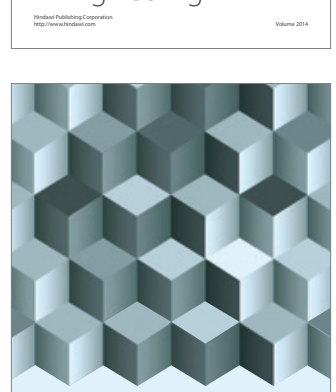

Journal of

Function Spaces
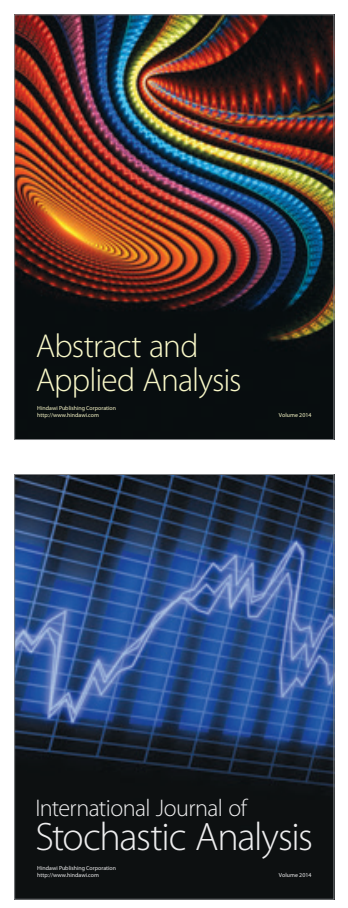

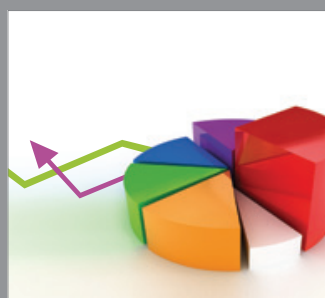

ournal of

Probability and Statistics

Promensencen
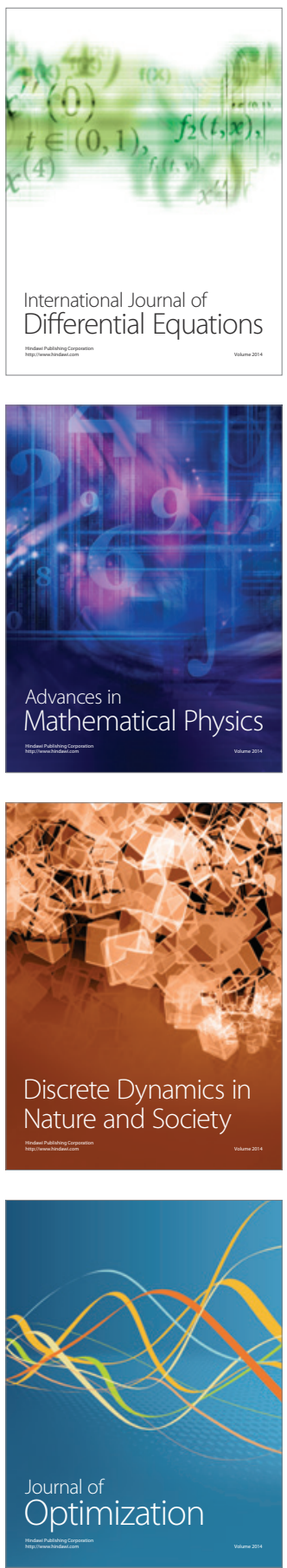